\title{
Variable optical attenuator fabricated by direct UV writing
}

Svalgaard, Mikael; Færch, Kjartan Ullitz; Andersen, L.U.

Published in:

Journal of Lightwave Technology

Link to article, DOI:

10.1109/JLT.2003.816889

Publication date:

2003

Document Version

Publisher's PDF, also known as Version of record

Link back to DTU Orbit

Citation (APA):

Svalgaard, M., Færch, K. U., \& Andersen, L. U. (2003). Variable optical attenuator fabricated by direct UV writing. Journal of Lightwave Technology, 21(9), 2097-2103. https://doi.org/10.1109/JLT.2003.816889

\section{General rights}

Copyright and moral rights for the publications made accessible in the public portal are retained by the authors and/or other copyright owners and it is a condition of accessing publications that users recognise and abide by the legal requirements associated with these rights.

- Users may download and print one copy of any publication from the public portal for the purpose of private study or research.

- You may not further distribute the material or use it for any profit-making activity or commercial gain

- You may freely distribute the URL identifying the publication in the public portal

If you believe that this document breaches copyright please contact us providing details, and we will remove access to the work immediately and investigate your claim 


\title{
Variable Optical Attenuator Fabricated by Direct UV Writing
}

\author{
Mikael Svalgaard, Kjartan Færch, and Lars-Ulrik Andersen
}

\begin{abstract}
It is demonstrated that direct ultraviolet writing of waveguides is a method suitable for mass production of compact variable optical attenuators with low insertion loss, low polarization-dependent loss, and high dynamic range. The fabrication setup is shown to be robust, providing good device performance over a period of many months without maintenance.
\end{abstract}

Index Terms-Attenuators, direct ultraviolet (UV) writing, integrated optics, waveguides.

\section{INTRODUCTION}

$\mathbf{S}$ ILICA-BASED integrated optical waveguide devices are key components in the deployment of high-speed optical telecommunication networks. The most successful methods for commercial fabrication of such devices currently combine a glass deposition technique such as plasma-enhanced chemical vapor deposition (PECVD) with photolithography and etching to define waveguide circuits. Such methods involve a large number of process steps and require a great deal of cleanroom processing. An alternative fabrication technique involves using a focused ultraviolet (UV) laser to write waveguides directly into the photosensitive core layer of a sample [1]. Since this method does not require photolithography or etching, the number of process steps is greatly reduced. In addition, the method does not require a cleanroom for UV writing. Consequently, direct UV writing is a technique with potentially much lower fabrication costs than the currently used techniques.

Basic integrated optical devices such as straight waveguides, s-bends, directional couplers, and 1:2 power splitters have been demonstrated with UV writing [2]-[4]. More complex devices, such as wavelength selective optical add-drop multiplexers [5], and waveguide lasers [6] have also been demonstrated with UV writing. However, it has not previously been investigated whether UV writing in terms of reproducibility, production capacity, and device performance is a viable industrial fabrication technique. We have addressed these questions by developing a setup that features a high degree of control over the UV writing process and by performing several test production runs of a commercially interesting device. The device chosen for this study was a variable optical attenuator (VOA) based on a Mach-Zehnder interferometer (MZI) with thermooptic phase shifters [7]. Although this device represents a fairly simple circuit layout, obtaining excellent performance on all key

Manuscript received February 6, 2003; revised May 30, 2003.

M. Svalgaard and K. Færch are with the Research Center COM, Technical University of Denmark, DK-2800 Lyngby, Denmark (e-mail: svlgrd@com.dtu. dk; kf@com.dtu.dk).

L.-U. Andersen is with the NKT Integration A/S, DK-3460 Birkerød, Denmark (e-mail: lua@nktintegration.com).

Digital Object Identifier 10.1109/JLT.2003.816889 parameters requires a high degree of process control due to the sensitive nature of the interferometer.

In this paper, we show that direct UV writing is capable of achieving a VOA performance that is similar to the best results obtained with etching/photolithography based silica-on-silicon technology. We also show that our UV writing setup provides this performance over an extended period of time without maintenance. The paper is concluded with an estimation of the production capacity of UV writing.

\section{EXPERIMENTAL PROCEDURE}

The samples used for this study consist of 4-in silicon wafers where the outer layer has been thermally oxidized. On top of this $\mathrm{SiO}_{2}$ layer, which serves as a buffer, a core-cladding structure was fabricated with PECVD. The thickness of the buffer/core/cladding is $16 / 5.5 / 12 \mu \mathrm{m}$. The core contains germanium and boron in a relative concentration so that the refractive index is matched (within $\pm 5 \times 10^{-4}$ ) to the surrounding layers [3]. Index matching the core layer enables the UV-written waveguides to exhibit a circular-mode profile and low coupling loss to standard telecom fiber [8]. Prior to UV exposure, the sample is loaded with molecular deuterium at a pressure of 190 bar until saturation to increase the photosensitivity [9].

Waveguides are fabricated by scanning the sample under a focused UV continuous-wave beam using high-precision $x-y$ translation stages. The optical layout of the experimental setup is shown in Fig. 1 and is similar to that used in previous work [10], [13]-[15], except for the fact that we now have extended the setup to automatically compensate for the slow drift of the UV beam direction and power. The UV beam has a wavelength of $257 \mathrm{~nm}$ and is generated with an intracavity-frequency-doubled argon-ion laser. The beam passes a computer controlled shutter so that it can be blocked when required in the waveguide writing process. Unfortunately, the direction and position of the UV beam in the horizontal plane may drift, depending on the tube current and thermal state of the laser. This effect leads to misalignment further down in the setup and is therefore compensated by means of a computer-controlled motorized mirror. The computer-controlled alignment is performed by directing with a mirror the beam to an angle and position sensor, which provides feedback to the computer. When the autoalignment is completed after roughly $1 \mathrm{~min}$, the mirror is moved out so that the beam can propagate to the remaining setup.

The beam is then deflected upwards and along an optical rail mounted across the sample. Here, the beam is spatially filtered and expanded, resulting in a circular-beam profile with a diameter at the first Airy minimum of $6 \mathrm{~mm}$. The Airy rings are removed with an iris diaphragm after which the beam is split so that 


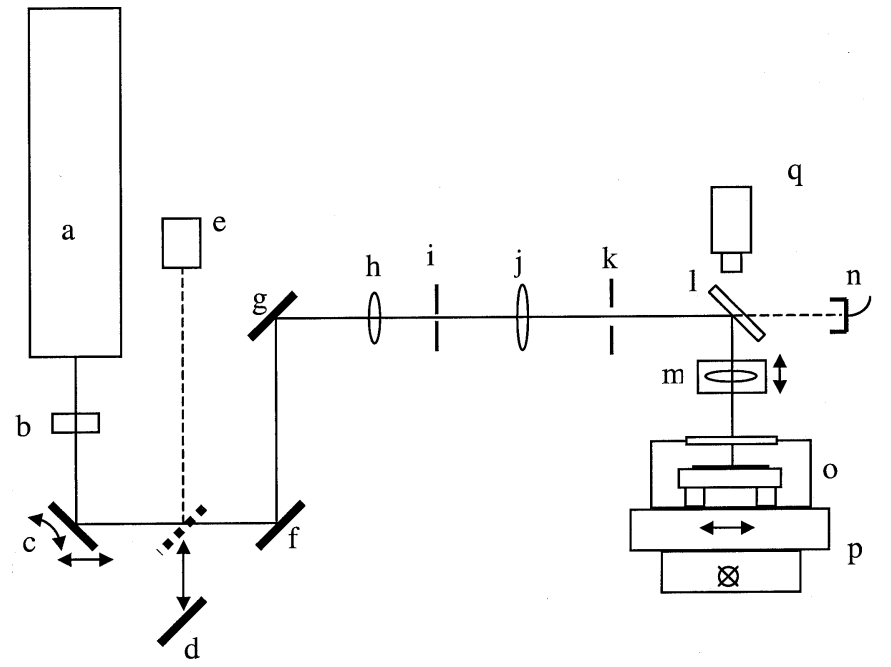

Fig. 1. Schematic drawing of the optical setup used for UV writing. The symbols represent the following components: a) UV laser; b) shutter; c) motorized mirror; d) movable mirror; e) angle and position sensor; f)-g) fixed mirrors; h)-j) beam expansion and spatial filtering; k) iris diaphragm; 1) 97:3 beam splitter; $\mathrm{m}$ ) focussing objective; n) Si photodiode; o) nitrogen-purged container; p) $x-y$ translation stages; and q) video camera.

$97 \%$ is reflected toward a focusing objective, which is mounted on a motorized holder. The objective has a focal length of $41 \mathrm{~mm}$ and creates a UV spot with a measured $1 / \mathrm{e}^{2}$ diameter of $3.1 \mu \mathrm{m}$ on the sample. The remaining $3 \%$ of the beam is transmitted to a Si p-i-n photodiode and used for continuous computer monitoring of the UV power during waveguide writing. If the UV power changes more than $\pm 0.5 \%$ from the nominal value, the computer pauses the UV writing and initiates an autoalignment sequence. Without such active monitoring and alignment, the incident power can drift by up to $20 \%$ during one run.

The sample is mounted in a nitrogen-purged container, which has an antireflection-coated silica window with a diameter of $100 \mathrm{~mm}$. The sample is thermoelectrically cooled to $-35^{\circ} \mathrm{C}$ to slow the rate of deuterium outdiffusion to negligible levels, thereby stabilizing the photosensitivity during UV writing [10]. Care is taken to ensure that the sample is level so that lateral motion does not introduce a defocusing of the UV beam. The sample chamber is mounted on dc current translation stages with an absolute accuracy of $0.1 \mu \mathrm{m}$ and a travel range of $100 \times 100 \mathrm{~mm}^{2}$. The stages are computer controlled and can perform accurate trajectory scans for velocities up to $\sim 500 \mu \mathrm{m} / \mathrm{s}$. A video camera is mounted above the beam splitter so that it provides a view of the writing process magnified by the objective. As mentioned subsequently, this camera is useful for aligning the UV spot accurately to any preexisting structures, for example, during UV trimming.

After UV writing, the sample is removed and annealed at $80{ }^{\circ} \mathrm{C}$ for $24 \mathrm{~h}$ to rapidly outdiffuse residual deuterium. The temperature is then increased to $150{ }^{\circ} \mathrm{C}$ for another $24 \mathrm{~h}$ to increase the thermal stability of the UV-induced index structures [11]. We have previously shown [12] that UV-written splitters, after a postfabrication anneal, exhibit no changes in performance after thousands of thermal cycles between room temperature and $80^{\circ} \mathrm{C}$.

The thermooptic phase shifters consist of gold electrodes deposited on the cladding above each arm of the interferometer.

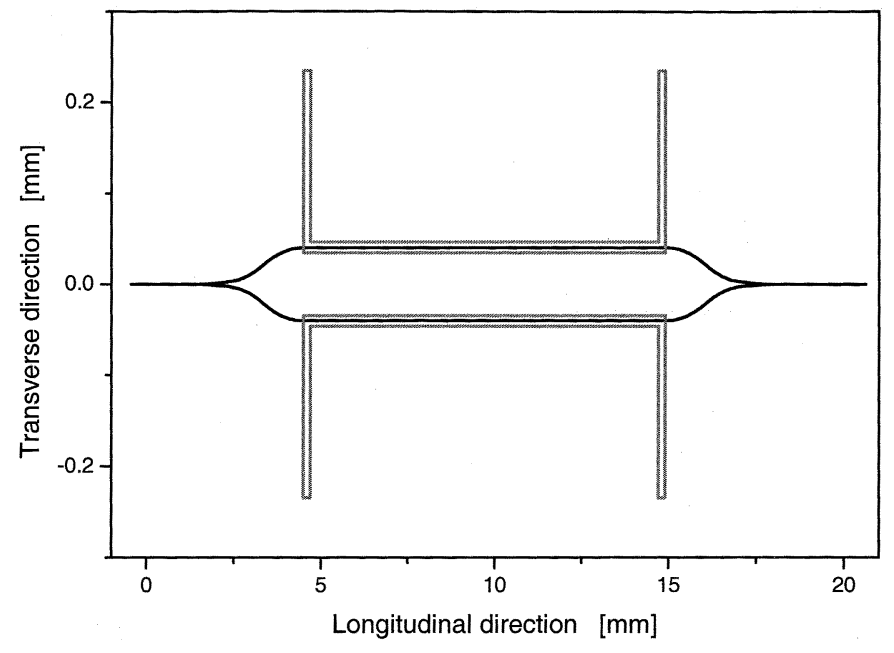

(a)

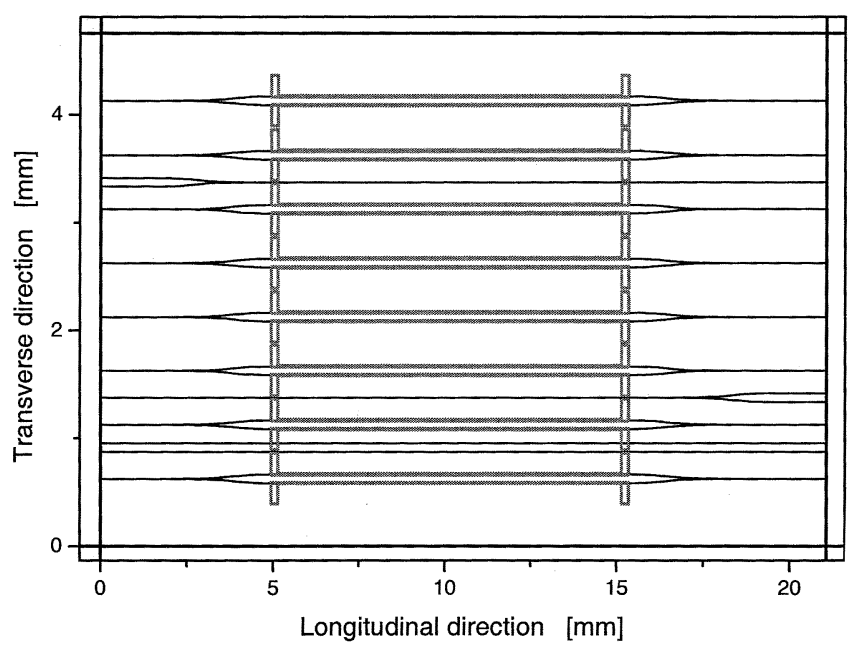

(b)

Fig. 2. (a) Close-up of the waveguide and electrode layout of a single VOA. (b) Overview of one multichannel VOA chip containing eight VOAs in parallel as well as two reference splitters and two reference waveguides.

The electrodes are fabricated using standard techniques of photolithography, evaporation, and liftoff. A 10-nm chromium layer is used under the gold for adhesion purposes. The thickness of the gold layer is $\sim 500 \mathrm{~nm}$, resulting in a resistance of $50-65 \Omega$, depending on the fabrication run. The electrode mask is aligned to the waveguide structure using either UV-written alignment marks or prefabricated aluminum alignment marks. The former technique is the most effective method, since it eliminates the additional wafer handling required for alignment mark fabrication. Finally, the wafer is diced out in individual chips.

\section{VOA DESIGN}

The layout of the VOA pursued in this work is shown in Fig. 2(a). Two $1 \times 2$ power splitters with an arm spacing of $80 \mu \mathrm{m}$ are connected to form an MZI. Thermooptic phase shifters (12 $\mu \mathrm{m}$ wide, $10 \mathrm{~mm}$ long) are located on the top cladding, directly above both arms of the interferometer. The electrode ends are terminated by $200 \times 200-\mu \mathrm{m}^{2}$ bonding pads. The total device length is $20 \mathrm{~mm}$.

Applying a voltage $U$ across one of the thermooptic shifters introduces a phase delay that determines the VOA transmission. 
By adding up normalized field amplitudes, the total insertion loss IL in decibels may be written as

$$
\begin{aligned}
\Pi= & \mathrm{IL}_{\mathrm{min}} \\
& -10 \mathrm{Log}\left(1-D \sin ^{2}\left(\frac{2 \pi}{\lambda}\left(L \delta n_{\mathrm{eff}}+L_{\mathrm{TO}} \delta n_{\mathrm{TO}}\right)\right)\right)
\end{aligned}
$$

where $\mathrm{IL}_{\min }$ is the minimum insertion loss, $D$ is a factor determining the dynamic range, $\lambda$ is the free-space wavelength, $L$ is the MZI arm length, $\delta n_{\text {eff }}$ is the difference in average effective index between the two arms, $L_{\mathrm{TO}}$ is the electrode length, and $\delta n_{\mathrm{TO}}$ is the thermooptically induced index difference between the arms. By assuming that the arm temperature difference is proportional to $U^{2} / R$, where $R$ is the electrode resistance, the thermooptically induced phase shift can be written as

$$
\delta n_{\mathrm{TO}}=\frac{\alpha U^{2}}{R}=\frac{\alpha U^{2}\left(1+\frac{\sigma U^{2}}{R_{0}}\right)}{R_{0}}
$$

where $\alpha$ is a proportionality constant. In the last expression, we have taken into account the temperature dependence of the resistance, where $\sigma$ is the thermal coefficient and $R_{0}$ is the room temperature resistivity. The dynamic range is the difference between the maximum and minimum transmission loss measured in decibels. By adding up normalized field amplitudes in the output arm, it may be shown that the dynamic range can be written as

$$
\begin{aligned}
& \text { dynamic range }[\mathrm{dB}] \\
& \qquad=-10 \log _{10}\left(\left(\frac{\sqrt{s_{1} s_{2}}-\sqrt{\left(1-s_{1}\right)\left(1-s_{2}\right)}}{\sqrt{s_{1} s_{2}}+\sqrt{\left(1-s_{1}\right)\left(1-s_{2}\right)}}\right)^{2}\right)
\end{aligned}
$$

where $s_{1}, s_{2}$ are the relative power fractions directed by the left and right splitter into adjoining paths. Hence, when $s_{1}=$ $1-s_{2}$ (point symmetrical configuration), the dynamic range would ideally be infinite. If one considers the loss of the VOA due to mismatching splitters $\left(s_{1} \neq s_{2}\right)$ in a similar manner as previously, it may be seen that only when $s_{1}=s_{2}=0.5$, do we get a device that does not radiate power into cladding modes at field recombination. Therefore, our goal is to utilize splitters that divide the signal equally into the interferometer arms.

Each splitter is written in three scans starting from the branching point and moving outwards, as described in [13]. The length of each splitter is roughly $3 \mathrm{~mm}$. The scan velocity used for the input waveguide and the first written output arm is $200 \mu \mathrm{m} / \mathrm{s}$. However, a slight reduction of the photosensitivity in the immediate surroundings of exposed areas leads to an asymmetric splitting ratio if the same scan velocity is applied in the second output arm [13]. Optimization showed that equal splitting was achieved when the scan velocity in the second arm was reduced to $70 \mu \mathrm{m} / \mathrm{s}$. Making the waveguides join in the middle of the interferometer is not a problem, since the stages have an accuracy of $0.1 \mu \mathrm{m}$. The MZI arm length as measured between the splitter branching points is $14.8 \mathrm{~mm}$. Each VOA requires six scans with a total processing time of $\sim 4 \mathrm{~min}$. One chip contains eight VOAs in parallel with a port spacing of
$500 \mu \mathrm{m}$, thereby forming a multichannel device, as shown in Fig. 2(b). In addition, two splitters and two straight waveguides are included as references. One 4-in wafer contains 38 chips.

A special feature of UV writing is that the processing time can be varied by roughly a factor of two to either side by adjusting the incident UV power. At a higher power, larger scan speeds can be applied to achieve a given waveguide width and index step. The upper limit is currently set by the fact that we cannot perform sufficiently accurate scans at velocities above $\sim 500 \mu \mathrm{m} / \mathrm{s}$. The chosen scan velocity of $200 \mu \mathrm{m} / \mathrm{s}$ is well below this upper limit. With 38 VOA chips per wafer, the total UV processing time becomes $23 \mathrm{~h}$, which would be well suited for a 24-h-based production schedule (allowing $1 \mathrm{~h}$ for wafer unloading and reloading).

\section{VOA PERFORMANCE}

In initial experiments, the UV power incident on the sample was adjusted to $45 \mathrm{~mW}$ so that a scan speed of $200 \mu \mathrm{m} / \mathrm{s}$ resulted in a $6.4-\mu \mathrm{m}$-wide waveguide. The waveguide birefringence was measured to be less than $5 \times 10^{-6}$ [14]. By comparing effective-index measurements and mode-field calculations, the index step was inferred to be $6.6 \times 10^{-3}$ [15]. These waveguide parameters provide excellent mode matching to a standard optical fiber (SMF-28).

Using these writing parameters, one complete wafer was UV processed, annealed, and equipped with electrodes according to the procedures given previously. The components were evaluated at a wavelength of $1.557 \mu \mathrm{m}$ using butt-coupled SMF-28 fibers and index matching oil for both input and output. The total $\mathrm{IL}$ and polarization-dependent loss (PDL) was measured with an EXFO IQ-3400 PDL/IL meter. For 38 reference waveguides, we measured an average total IL of $0.53 \mathrm{~dB}$ and an average PDL of $0.26 \mathrm{~dB}$. The data scattering corresponded to a standard deviation of $0.03 \mathrm{~dB}$, which is equal to the specified accuracy of the IL/PDL meter used for the measurements.

Loss measurements of 38 reference splitters showed that the average splitting ratio was $0.51(2.9 \mathrm{~dB})$. This is in excellent agreement with the optimization performed several weeks earlier and exemplifies the stability of the fabrication setup. The splitter excess loss, as measured relative to a reference waveguide, was $0.05 \mathrm{~dB}$ with a standard deviation of $0.05 \mathrm{~dB}$. Hence, the splitters have very low excess loss and will not contribute significantly to the overall loss of the VOA. Finally, the broad-band response was measured with a white-light source and an optical spectrum analyzer. As expected for this kind of device, the splitting ratio was flat to within $\pm 0.3 \mathrm{~dB}$ from 1300-1650 nm.

Visual inspection under a microscope showed that the VOA electrodes were misaligned to the waveguides by $\sim 3 \mu \mathrm{m}$, which can probably be improved significantly by further practice. The alignment tolerance was later investigated by deliberately misaligning the electrodes by up to $\pm 6 \mu \mathrm{m}$ without any significant change in performance. This tolerance may be caused by lateral dispersion of the applied heat. Electrical contact to the bonding pads was achieved with tungsten needles mounted on $x-y-z$ probe mounts. Electrical contact could also be achieved by permanently bonding a gold wire to the electrode pads. 


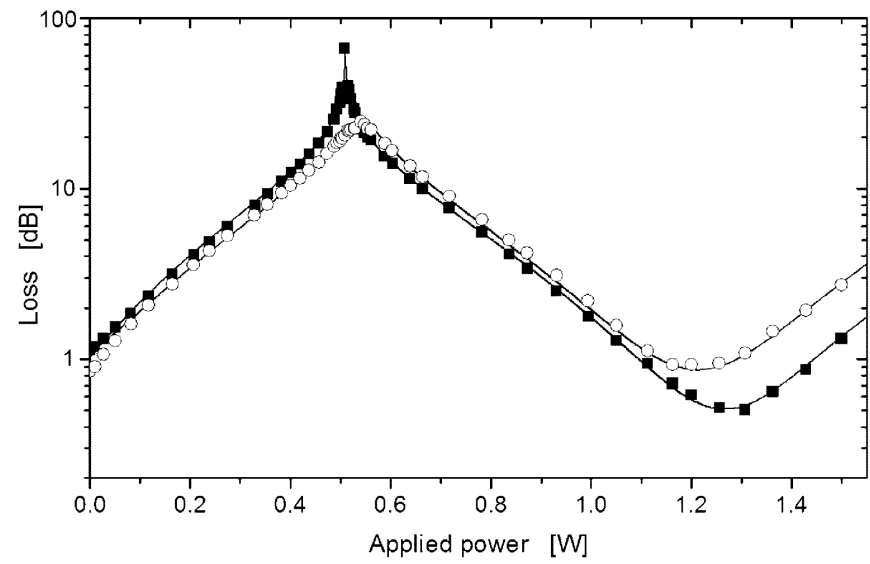

Fig. 3. VOA loss curves versus the power applied to one electrode (squares: TE mode; circles: TM mode). These curves were measured before UV trimming. The solid lines for each data set represent fits using the theoretically expected behavior (1).

The loss characteristics of a randomly selected VOA were measured for transverse electric (TE)- and transverse magnetic (TM)-polarized light, which corresponded to the extremes of the polarization-dependent response. The resulting loss curves versus the applied power $\left(U^{2} / R\right)$ are shown in Fig. 3. When no power is applied, the loss is $\sim 1 \mathrm{~dB}$. As power is applied, the losses increase gradually, reaching peak values for $\sim 0.5 \mathrm{~W}$. For this particular VOA, the TE polarization exhibits a very high peak loss of $\sim 70 \mathrm{~dB}$, while the TM- polarization has a more moderate peak value of $25 \mathrm{~dB}$. As the applied power is increased further, the losses decrease, reaching a minimum value of $0.5 \mathrm{~dB}$ for the TE polarization and $0.8 \mathrm{~dB}$ for the TM-polarization. The minimum losses are achieved for an applied power of $\sim 1.2 \mathrm{~W}$ and are just $0.1-0.2 \mathrm{~dB}$ higher than the insertion loss of a straight reference waveguide. The power required for a $\pi$ phase shift is $0.65 \mathrm{~W}$. With a larger buffer layer thickness and by etching heat-insulating grooves around the heaters, it is possible to reduce this power requirement by roughly an order of magnitude [16]. The loss curves show that the interferometer with no power applied is unbalanced by $\sim 0.1 \mathrm{rad}$, in spite of our attempts to perform the UV writing identically for the two arms. This unbalance may be caused by a slight drift in the UV power during the fabrication process and can be counterbalanced by applying a corresponding bias on the other electrode. The theoretically expected loss curves, as given in (1) and (2), were fitted to the measured data set for each polarization. The result is shown in Fig. 3 as solid lines, showing a very good agreement with the actual measurements.

A total of 23 other VOAs from the wafer were selected randomly for characterization, with overall results similar to those given previously. The dynamic range for the TE polarization was on average $31 \mathrm{~dB}$, while the corresponding value for the TM polarization was $34 \mathrm{~dB}$. Hence, the VOA from Fig. 3 is somewhat atypical, since it has a much higher dynamic range for the TE polarization. The typical device-to-device scattering in dynamic range due to fabrication imperfections was roughly $\pm 3 \mathrm{~dB}$. The performance based on all 24 measured VOAs is summarized in Table I, including the observed scatter due to fabrication imperfections.
TABLE I

VOA Performance Summary Based on Measurements of 24 DeVices From a Single Production Run; the LAST Column INDICATES the ObServed Device-to-Device Performance Scatter (Roughly Two Standard Deviations) Due to FABRICATION IMPERFECTIONS

$\begin{array}{lll}\text { Chip size } & 21 \times 4.8 \mathrm{~mm}^{2} & \\ \text { Number of channels } & 8 & \\ \text { Minimum insertion loss }^{1} & 0.7 \mathrm{~dB} & \pm 0.1 \mathrm{~dB} \\ \text { Dynamic range }^{1} & 33 \mathrm{~dB} & \pm 3 \mathrm{~dB} \\ \text { PDL @ 0 dB attenuation } & 0.2 \mathrm{~dB} & \pm 0.1 \mathrm{~dB} \\ \text { PDL @ 10 dB attenuation } & 2 & \pm 0.2 \mathrm{~dB} \\ \text { PDL @ 20 dB attenuation }^{2} & 0.6 \mathrm{~dB} & \pm 0.2 \mathrm{~dB} \\ \text { Power requirement }^{3} & 1.2 \mathrm{~dB} & \pm 50 \mathrm{~mW} \\ \text { Spectral range }^{2} & 630 \mathrm{~mW} & \\ \text { Spectral flatness }^{4} & 1525-1570 \mathrm{~nm} & \end{array}$

1: polarization averaged

2: after UV trimming

3: for a $\pi$ phase shift

4: at $10 \mathrm{~dB}$ attenuation

From Fig. 3, it is apparent that the loss curves for the TE and TM polarization differ somewhat. The absolute difference (PDL) increases for higher loss values, reaching a value of $1 \mathrm{~dB}$ for an attenuation of $\sim 7 \mathrm{~dB}$, which is unacceptable for most applications. In the following section, means for reducing the PDL will be discussed.

\section{REDUCTION OF PDL}

The PDL evident in Fig. 3 arises due to differences in horizontal and vertical scaling and different horizontal offset between the TE and TM modes. Each of these contributions is discussed in the following subsections.

\section{A. Horizontal Scaling}

This effect is evident in Fig. 3 as a different thermooptic efficiency for the TE and TM polarizations; in our case, the difference is $\sim 8 \%$. It arises due to a birefringent component in the phase shift induced by the thermooptic heaters [17]. When heated, the glass is restricted from expanding in the horizontal direction, which leads to an anisotropic stress distribution. The larger the heating, the greater this effect will be.

\section{B. Vertical Scaling}

In Fig. 3, it is seen that the dynamic range for the TE and TM modes are different. The difference is much less pronounced in most of the other VOAs that were characterized. From (3), it is seen that such behavior can arise due to a polarization-dependent splitting ratio. 


\section{Horizontal Offset}

For zero applied power, it is apparent that there is an offset of the TE and TM curves in Fig. 3. From (1), it follows that such an offset can arise due to a difference in waveguide birefringence between the interferometer arms (birefringence imbalance). This gives rise to a polarization-dependent phase shift: $\left(B_{1}-B_{2}\right) \cdot 2 \pi L / \lambda$, where $B_{i}=n_{\text {eff. } i}^{\mathrm{TE}}-n_{\text {eff. } i}^{\mathrm{TM}}$ is the birefringence for interferometer arm $i$. For the device in Fig. 3, the deduced birefringence imbalance is $\sim 3 \times 10^{-6}$. Since $L$ is many wavelengths long, even such a low value may lead to significant PDL, especially near the attenuation peaks.

These PDL governing effects are not specifically associated with the UV writing fabrication technique. Instead, they are related to the MZI-based VOA design, which is very sensitive to any phase imbalance due to the long arm length. Another contributing factor is the silica-on-silicon material system, which necessitates the use of thermooptic phase shifters and has inherent stress problems due to the mismatch between glass and silicon thermal expansion coefficients.

In this project, we have concentrated on minimizing PDL due to phase shifter birefringence, since this effect is the dominating PDL source for intermediate attenuation values. The most effective way of reducing this parameter is to etch stress-releasing grooves on both sides of the electrodes [17]. This also lowers the lateral heat dispersion, resulting in a lower power consumption and faster response time [16]. Unfortunately, in our experiments, we did not have access to this option. Instead, we have used phase trimming with UV light to obtain a significant PDL reduction. This is achieved by inducing a permanent phase shift so that the attenuation peaks occur for lower applied power where the phase shifter birefringence is less pronounced.

A series of UV trimming experiments utilizing the waveguide writing setup were carried out to test this method of PDL reduction. Alignment to the waveguide pattern was quite easy, since the waveguides emit a bright-blue luminescence under UV exposure. An incident UV power of $50 \mathrm{~mW}$ was used with a spot size of $8 \mu \mathrm{m}$ (to uniformly cover one waveguide) and a scan velocity of $80 \mu \mathrm{m} / \mathrm{s}$. By performing a series of scans with varying lengths, we determined that the trimming resulted in a reproducible effective index change of $3 \times 10^{-4}$. After UV trimming, the sample was annealed at $150{ }^{\circ} \mathrm{C}$ for $12 \mathrm{~h}$ to minimize thermal instability of the induced phase shift when operating the phase shifters. The trimming was carried out on one interferometer arm between a splitter branching point and the gold electrode. To avoid changing the splitting ratio, the trimming should not be carried out too closely to the branching point. We chose to start at a point where the interarm distance was $23 \mu \mathrm{m}$ and saw no changes in the VOA dynamic range or excess loss, as would result if the splitting ratio had been changed. The VOA from Fig. 3 was trimmed so that the attenuation peaks were moved from an applied power of 500 down to $70 \mathrm{~mW}$, as illustrated in Fig. 4. This corresponded to a trimmed length of $900 \mu \mathrm{m}$ on both splitters, a process that lasted $23 \mathrm{~s}$. The data suggests that the minimum loss is actually lowered slightly by the UV trimming; however, we believe that this is merely a measurement-induced offset, since it did not consistently appear for other trimmed devices. It is seen that the polarization-dependent phase shift at the attenuation peaks is much smaller than before. By fitting (1) to the measured data, it may be shown that

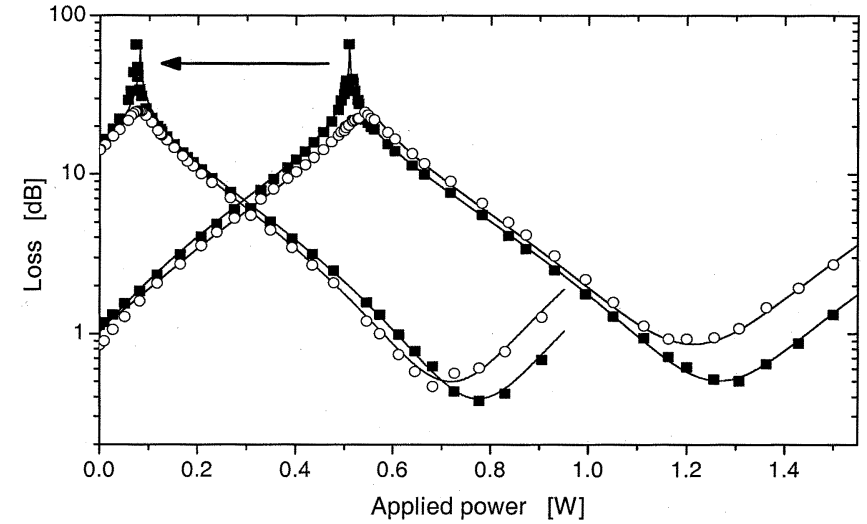

Fig. 4. VOA loss curves versus the power applied to one electrode (squares: TE mode; circles: TM mode). The change due to UV trimming is illustrated by the arrow.

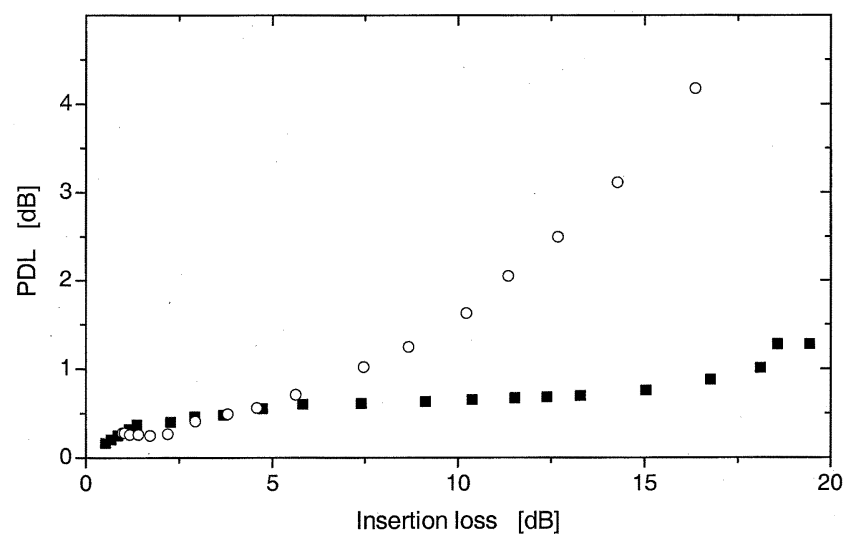

Fig. 5. Measured VOA PDL versus the attenuation level (circles: before UV trimming; squares: after UV trimming).

the birefringence imbalance has also been reduced by at least an order of magnitude to $<3 \times 10^{-7}$ (the estimated fitting accuracy). This additional effect of UV trimming further reduces the PDL; however, it was not anticipated and is not fully understood at this time. The PDL versus attenuation is plotted in Fig. 5 before and after UV trimming. Three other VOAs were also UV trimmed, with similar results. After UV trimming, the PDL is typically below $1.2 \mathrm{~dB}$ for an attenuation of $20 \mathrm{~dB}$ (summarized in Table I). For low attenuation levels, the trimming may lead to increased PDL, as evident in Fig. 5. However, the increase is only $\sim 0.1 \mathrm{~dB}$, which may be acceptable in light of the much larger PDL reduction at higher attenuation levels. Even though we have demonstrated significant PDL reduction with UV trimming, we consider stress-releasing grooves to be a more cost-effective solution for large-scale production, because it does not require measurement of the MZI imbalance and because it also lowers the power requirements [16].

\section{SPECTRAL RESPONSE}

From (1), it is seen that the VOA spectral response is mainly governed by the $1 / \lambda$ term in the phase-shift expression. Secondary effects include dispersion of the phase imbalance and of the thermooptic coefficient. From (3), for the dynamic range, we see that a wavelength-dependent splitting ratio can also influence the VOA spectral response. However, since our splitters 


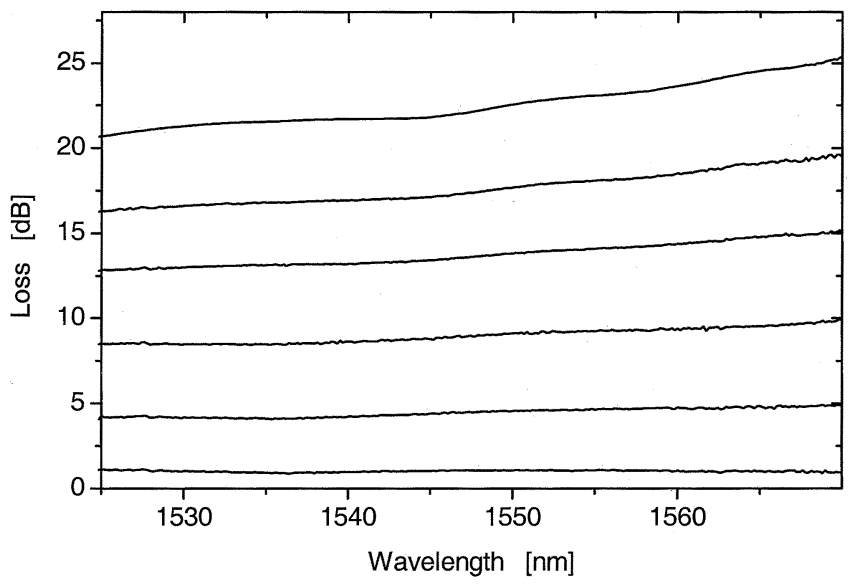

Fig. 6. Measured VOA spectral variation for various attenuation levels.

have a very flat spectral response, it may be shown that this effect is negligible.

From (1), it is seen that the peak spectral variation over the $C$ band $(1525-1570 \mathrm{~nm})$ for all phase retardations increases with the dynamic range. The value of this variation due to the $1 / \lambda$ term alone is $\pm 0.4 \mathrm{~dB}$ for a $10-\mathrm{dB}$ dynamic range and $\pm 1 \mathrm{~dB}$ for a 20-dB dynamic range. In actual VOA components, the spectral response over the $C$ band has been measured using a polarized amplified spontaneous emission (ASE) source. A typical measurement for one polarization is shown in Fig. 6 at various attenuation levels. The spectral variation is very flat $( \pm 0.2 \mathrm{~dB})$ for low attenuations of a few decibels. For an attenuation of $10 \mathrm{~dB}$, the spectral variation has increased to $\pm 0.8 \mathrm{~dB}$, while for $20 \mathrm{~dB}$ attenuation, the variation is $\pm 2 \mathrm{~dB}$. This is roughly a factor of two larger than calculated for the $1 / \lambda$ term alone in (1). We speculate that the excess spectral variation is due to dispersion of the phase imbalance and/or of the thermooptic coefficient.

\section{THERMAL STABILITY}

The finite thermal stability of UV-induced index changes leads to general concerns about the long-term stability of UVwritten components, especially sensitive devices employing interferometers and thermal heaters. We have therefore subjected our VOA components to temperatures up to $85^{\circ} \mathrm{C}$ for several days; however, no changes in performance could be detected. This is not surprising, since the structure is annealed uniformly. We also left one electrode running at $1-\mathrm{W}$ power for 12,24 , and $72 \mathrm{~h}$, thereby producing a prolonged localized heating. After $12 \mathrm{~h}$, a drift of the attenuation peak locations of $\sim 30 \mathrm{~mW}$ $(0.15 \mathrm{rad})$ had occurred. The drift between 24 and $72 \mathrm{~h}$ was roughly five times smaller and just barely measurable. This behavior shows that either a higher postfabrication annealing temperature or a burn-in of the electrodes could be employed in future applications to greatly reduce this instability.

\section{FABRICATION STABILITY}

Based on the performance reported in the previous sections, we conclude that UV writing yields a VOA performance that is comparable to that obtained with more widely used silica-on-silicon fabrication techniques. However, for an industrial implementation of UV writing, such a performance must be achiev-
TABLE II

Summarized VOA Performance From Four Production RunS; THE LAST Two Runs INCLUdED A MinOR CHANGE IN THE MACH-ZEHNDER INTERFEROMETER DESIGN WHICH REDUCED THE MINIMUM INSERTION LOSS AND INCREASED THE DYNAMIC RANGE

$\begin{array}{lcccc}\text { Production run \# } & 1 & 2 & 3 & 4 \\ \text { Sampled number of VOA's } & 14 & 16 & 24 & 11 \\ \text { Minimum insertion loss [dB] } & 1.3 & 1.4 & 0.7 & 0.9 \\ \text { Dynamic range [dB] } & 23 & 23 & 33 & 32 \\ \text { PDL @ 0 dB attenuation } & 0.2 & 0.3 & 0.2 & 0.3 \\ \text { PDL @ 20 dB attenuation }{ }^{1} & 1.1 & 1.3 & 1.2 & 1.2 \\ \text { Power requirement } & 650 & 660 & 630 & 670\end{array}$

1: after UV trimming

able on a routine basis over an extended period of time. To test whether this is the case, we have conducted a series of four UV writing production runs over a period of $9 \mathrm{mo}$. In this time period, no maintenance or manual alignment of the laser or UV writing setup was performed. In addition, the setup was often used for other research projects. The VOA fabrication process and subsequent measurements are identical to that described previously. In Table II, we list for each production run, the number of characterized VOAs and the average value of the minimum insertion loss, dynamic range, PDL, and power consumption. The observed scattering in performance within each production run was similar to that reported earlier in this paper. In the first two production runs, the average VOA minimum loss was $\sim 1.3 \mathrm{~dB}$, which was $\sim 0.5 \mathrm{~dB}$ larger than that of a straight reference waveguide. In addition, the average dynamic range was only $23 \mathrm{~dB}$. This prompted us to optimize how the waveguides were joined face to face in the MZI. Instead of overlapping the waveguide scans by $100 \mu \mathrm{m}$, the layout was changed so that the waveguides stopped a few micrometers short from each other. This minor change resulted in the reduced loss and higher dynamic range seen for the last two production runs. The PDL problems inherent to this design were seen consistently, and UV trimming was applied on roughly one quarter of all measured devices, resulting in the PDL values given in the table. This shows that UV trimming is a reliable tool for reducing the PDL. Finally, the VOA power consumption was very stable throughout all four production runs, which is not surprising due to the insensitivity toward alignment caused by lateral heat dispersion.

Taking the minor change in scanning layout into account, the observed variations between each production run are very low. These results indicate that UV writing holds promise as a method for accurate and reliable production of silica-on-silicon optical devices. It should be emphasized that this conclusion is for components of a complexity similar to the VOA design used here. We have not shown that UV writing currently possesses 
the ability for reliable production of more complex components, such as arrayed-waveguide gratings or tunable dispersion compensators.

Under the circumstances implemented here, the UV writing process requires roughly $50 \mathrm{~mW}$ of power. Since the UV laser (Sabre FreD, Coherent, Inc.) is rated for 1-W output, it should be possible to operate multiple identical writing setups in parallel. In addition, multiple wafers can be mounted on one set of translation stages. Based on our experience, we estimate that one laser could be used to power two writing setups, each with three wafers. In this estimation, the required power (including that lost in the UV optics) would be $\sim 50 \%$ of the specified laser output power. Such a system could process six wafers/d, each containing 38 multichannel VOA chips, as used in this study. Hence, the potential production capacity of a setup based on a single UV laser is in the order of $10^{4}-10^{5}$ multichannel VOA chips/y.

\section{CONCLUSION}

In this paper, we have described the fabrication of variable optical attenuators by direct UV writing. In order to obtain a high degree of control and reproducibility, a laser writing setup with computer-controlled beam alignment and monitoring of the incident beam power has been developed. The chosen VOA design is based on $1 \times 2$ splitters forming an MZI with gold electrodes mounted along the interferometer arms to provide an electrically controlled phase shift. The performance achieved hereby was similar to the best results obtained previously with other silica-based fabrication methods. Based on a large number of measured components, we observed a VOA minimum insertion loss of $\sim 0.7 \mathrm{~dB}$, while the dynamic attenuation range exceeded $30 \mathrm{~dB}$. Initially, the VOA PDL was unacceptably high, mainly due to a birefringent component in the phase shift induced by the thermooptic heaters. We have shown that UV trimming can be applied to reduce this effect, leading to a VOA PDL of $\sim 1 \mathrm{~dB}$ at $20-\mathrm{dB}$ attenuation. The spectral flatness over the $C$ band at $10-\mathrm{dB}$ attenuation was measured to be $\pm 0.8 \mathrm{~dB}$. The device-to-device scattering in VOA performance within one wafer was minimal and should not significantly limit the production yield.

To test direct UV writing as an industrial fabrication technique, we have performed VOA production runs over a period of several months, during which the fabrication setup was not maintained or aligned manually. The wafer-to-wafer average performance variations were smaller than the variations seen within a single wafer; hence, the UV writing technique provides a very stable platform for continuous production over extended periods of time with little or no need for adjustments. From the results presented in this work, it is estimated that the production capacity of one UV laser is roughly $10^{4}-10^{5}$ multichannel VOA chips/y.

\section{REFERENCES}

[1] M. Svalgaard, C. V. Poulsen, A. Bjarklev, and O. Poulsen, "Direct UV-writing of buried single-mode channel waveguides in Ge-doped silica films," Electron. Lett., vol. 30, pp. 1401-1402, 1994.

[2] M. Svalgaard and M. Kristensen, "Directly UV written silica-on-silicon planar waveguides with low loss," Electron. Lett., vol. 33, pp. 861-862, 1997.

[3] G. D. Maxwell and B. J. Ainslie, "Demonstration of a directly written directional coupler using UV induced photosensitivity in a planar silica waveguide," Electron. Lett., vol. 31, pp. 95-96, 1995.

[4] M. Svalgaard, "Direct writing of planar waveguide power splitters and directional couplers using a focused ultraviolet laser beam," Electron. Lett., vol. 33, pp. 1694-1695, 1997.

[5] M. Y. Park, W. Yoon, S. Han, and G. Hugh Song, "Fabrication of low-cost planar wavelength-selective optical add-drop multiplexer by employing UV photosensitivity," Electron. Lett., vol. 38, pp. $1532-1533,2002$.

[6] C. B. E. Gawith, A. Fu, T. Bhutta, P. Hua, D. P. Shepherd, E. R. Taylor, and P. G. R. Smith, "Direct-UV-written buried channel waveguide lasers in direct-bonded intersubstrate ion-exchanged neodymium-doped germano-borosilicate glass," Appl. Phys. Lett., vol. 81, pp. 3522-3524, 2002 .

[7] T. Kawai, M. Koga, M. Okuno, and T. Kitoh, "PLC type compact variable optical attenuator for photonic transport network," Electron. Lett., vol. 34, pp. 264-265, 1998.

[8] D. Zauner, K. Kulstad, J. Rathje, and M. Svalgaard, "Directly UV written silica-on-silicon planar waveguides with low insertion loss," Electron. Lett., vol. 34, pp. 1582-1584, 1998.

[9] P. J. Lemaire, R. M. Atkins, V. Mizrahi, and W. A. Reed, "High pressure $\mathrm{H}_{2}$ loading as a technique for achieving ultrahigh UV photosensitivity and thermal sensitivity in $\mathrm{GeO}_{2}$ doped optical fibers," Electron. Lett., vol. 29, pp. 1191-1193, 1993.

[10] M. Svalgaard, "Effect of $\mathrm{D}_{2}$ outdiffusion on direct UV writing of waveguides," Electron. Lett., vol. 35, pp. 1840-1841, 1999.

[11] T. Erdogan, V. Mizrahi, P. J. Lemaire, and D. Monroe, "Decay of ultraviolet-light induced Bragg gratings," J. Appl. Phys., vol. 76, pp. 73-80, 1994.

[12] K. Kulstad and M. Svalgaard, "Thermal stability of directly UV-written waveguides and devices," in Proc. Eur. Conf. Optical Communication, Madrid, Spain, Sept. 20-24, 1998, Paper MoA03, pp. 33-34.

[13] K. Færch and M. Svalgaard, "Symmetrical waveguide devices fabricated by direct UV writing," IEEE Photon. Technol. Lett., vol. 14, pp. 173-175, Feb. 2002.

[14] M. Svalgaard, "Zero-birefringence UV written waveguides," in Tech. Dig. Series OSA Topical Meeting Bragg Gratings, Photosensitivity, Poling, vol. 33, 1999, pp. 283-285.

[15] L. Leick, A. Harpøth, and M. Svalgaard, "Empirical model for the waveguiding properties of directly UV-written waveguides," Appl. Opt., vol. 41, pp. 4325-4330, 2002

[16] S. Sohma, T. Goh, H. Okazaki, M. Okuno, and A. Sugita, "Low switching power silica-based super high delta thermo-optic switch with heat insulating grooves," Electron. Lett., vol. 38, pp. 127-128, 2002.

[17] Y. Inoue, K. Katoh, and K. Masao, "Polarization sensitivity of a silica waveguide thermooptic phase shifter for planar lightwave circuits," IEEE Photon. Technol. Lett., vol. 4, pp. 36-38, Jan. 1992.

Mikael Svalgaard, photograph and biography not available at the time of publication.

Kjartan Færch, photograph and biography not available at the time of publication.

Lars-Ulrik Andersen, photograph and biography not available at the time of publication. 